\title{
O WhatsApp e as Aprendizagens Significativa e Colaborativa
}

Sergio da Costa Nunes - IFSul - sergio.dacostanunes@ gmail.com - 0000-0001-8276-5004

Rosane Aragón - UFRGS - rosane.aragon@gmail.com - 0000-0002-0307-4457

\section{Resumo}

Esta pesquisa apresenta um estudo qualitativo, desenvolvido em uma turma de alunos de um curso superior, da área de informática, objetivando explorar as potencialidades das tecnologias digitais colaborativas nos processos de ensino e aprendizagem. Para tanto, utilizou-se o aplicativo de mensagens WhatsApp como interface pedagógica para o desenvolvimento de um trabalho na disciplina de Sistemas de Informação. A teoria da aprendizagem significativa foi articulada com a aprendizagem colaborativa para evidenciar os processos ativos de aprendizagem. Para a aplicação do trabalho, dividiu-se a turma em quatro grupos de cinco componentes e aplicou-se um questionário, o qual fora extraído de um artigo com conteúdo específico da disciplina. Mapas conceituais foram utilizados como ferramenta pedagógica para verificar a aprendizagem significativa durante o decurso da pesquisa, propiciando a análise dos subsunçores organizados pelos alunos durante a elaboração das respostas. A análise dos subsunçores apontou resultados relevantes quanto à utilização do WhatsApp para os processos de ensino e aprendizagem, estimulando os pesquisadores a novos estudos.

Palavras-chave: Aprendizagem significativa, Aprendizagem colaborativa, WhatsApp.

\section{WhatsApp and Meaningful and Collaborative Learning}

\begin{abstract}
This research presents a qualitative study, developed in a class of students in a college course in the area of information technology, aiming to explore the potential of collaborative digital technologies in teaching and learning processes. To this end, the messaging application WhatsApp was used as a pedagogical interface for the development of an assignment in the subject Information Systems. The theory of meaningful learning was articulated with collaborative learning to highlight the active learning processes. For the application of the work, the class was divided into four groups of five components and a questionnaire was applied, which was extracted from an article with specific content of the discipline. Concept maps were used as a pedagogical tool to verify the significant learning during the course of the research, providing the analysis of the subsumers organized by the students during the elaboration of the answers. The analysis of the subsumers indicated relevant results regarding the use of WhatsApp for the teaching and learning processes, stimulating the researchers to new studies.
\end{abstract}

Keywords: Meaningful learning, Collaborative learning, WhatsApp.

\section{Introdução}

As tecnologias móveis propiciaram o surgimento de novos modelos educacionais, procurando atender uma geração cada vez mais conectada ao mundo virtual. Um desses modelos é a chamada mobile learning, que utiliza ferramentas digitais portáteis como interface V. $19 \mathrm{~N}^{\mathrm{o}} 2$, Dezembro, 2021 RENOTE 
para auxiliar na aprendizagem. Conforme Kurtz et al (2015), a característica do mobile learning é trazer mobilidade e conectividade, aumentando a usabilidade e, consequentemente, o aprendizado.

A mobile learning pode ser evidenciada através dos estudos de Smit \& Goede (2012), nos quais demonstraram que a utilização de plataformas de mensagens como o WhatsApp possui bom potencial para a aprendizagem, tornando os alunos mais ativos e motivados em seus estudos. É pertinente destacar que essas características da mobile learning estão associadas com a chamada aprendizagem ativa.

Conceitualmente, a aprendizagem ativa estabelece o aluno como sujeito na construção do seu conhecimento, em oposição às metodologias tradicionais, que consideram o professor como elemento central dos processos de ensino e aprendizagem. Já na aprendizagem ativa, o aluno é mais participativo, colaborativo, enfim, mais ativo, aparecendo o professor como organizador ou condutor da aprendizagem (Santos e Ferrari, 2017).

De acordo com Mill (2021), a aprendizagem ativa refere-se a um conjunto de práticas pedagógicas que considera o estudante como protagonista do próprio processo de construção do conhecimento. Neste contexto, considera-se a realidade socioambiental do aluno como elemento essencial no estímulo de sua aprendizagem, desta forma, os conteúdos e metodologias devem preferencialmente proverem essa característica. Mattar (2017) elenca algumas metodologias ativas que contribuem significativamente para a construção de uma aprendizagem ativa: aprendizagem híbrida, sala de aula invertida, gameficação, mapas conceituais, etc..

Assim, pode-se considerar que a aprendizagem ativa está respaldada pela aprendizagem significativa porque, conforme Mill (2021), o objetivo da proposição de abordagens ativas é que o estudante aprenda de modo a encontrar significado no que estuda. Ausubel et al (1980) estabelece que a aprendizagem significativa ocorre quando uma nova informação é relacionada de maneira substantiva e não arbitrária, a um aspecto relevante, previamente existente na estrutura cognitiva do estudante. Nesta mesma linha, Novak (2005) define-a como a relação que deve existir entre os novos conhecimentos e os conhecimentos prévios ou subsunçores existentes na estrutura cognitiva do aluno. Sendo assim, o que o aluno já sabe é fundamental para que a aprendizagem de novos conteúdos adquira significado para ele, em um processo que Ausubel et al (1980) define como "ancoragem", em que conhecimentos anteriores irão suportar os novos conhecimentos.

Partindo desses conceitos, pode-se evidenciar a importância do ambiente e das vivências do aluno como fatores que influenciam na formação de seus subsunçores, pois, de acordo com Moreira (2011), para que a educação faça sentido, o conhecimento precisa ser construído pelos alunos, de acordo com suas realidades e onde estão inseridos. Sendo assim, o aluno deve ser protagonista na construção de sua aprendizagem, as aulas expositivas pouco ou quase nada contribuem, porque para um conhecimento tornar-se significativo para o aluno os processos de ensino e aprendizagem devem estar baseados nas interpretações e interações socioculturais da sua realidade (Mill, 2021). Desta forma, a aprendizagem deve ser significativa tanto para o professor quanto para o aluno, o que conduz para as relações entre as aprendizagens ativa e significativa.

Os pressupostos das aprendizagens ativa e significativa, se bem estruturados, podem contribuir para a mobile learning utilizando o WhatsApp como interface virtual para que os estudantes possam interagir para a consecução de seus estudos. Nesse contexto, este artigo apresenta um estudo qualitativo feito em uma turma de alunos, de um curso superior da área de Informática, na disciplina de Sistemas de Informação, com o conteúdo de ERP - Enterprise Resource Planning, desenvolvido com a utilização de mobiles learnings em conjunto com o WhatsApp. O estudo evidenciou a aprendizagem ativa dos estudantes através de seus atributos significativos, utilizando os mapas conceituais como ferramenta de avaliação e os links cognitivos como parâmetros de medida. 
Para isso, este artigo está organizado em cinco seções, sendo a primeira esta introdução, que apresenta a mobile learning e o aplicativo de mensagens WhatsApp como ferramentas do processo de ensino e as aprendizagens ativa e significativa, como potenciais indutoras deste processo. Na segunda parte, apresenta-se o conceito e relações da aprendizagem colaborativa com o aplicativo Whatsapp. Na terceira parte, encontram-se os conceitos de mapas conceituais e links cognitivos, utilizados para estabelecer os parâmetros avaliativos para referendar o estudo. Após, na quarta parte, tem-se a metodologia, com as características dos grupos pesquisados e as abordagens utilizadas na pesquisa. Na quinta seção, apresentam-se os resultados e as discussões elaboradas com os mapas conceituais e os subsunçores organizados e, finalmente, na sexta etapa chega-se às conclusões a respeito da pesquisa, seguidas, obviamente, pelas referências que embasaram o estudo.

\section{Aprendizagem Colaborativa}

De acordo com Laal (2012), a aprendizagem colaborativa é uma abordagem para os processos de ensino e aprendizagem, em que os alunos trabalham em grupos objetivando a resolução de problemas ou realizar tarefas, sendo assim, o conhecimento gerado pelos membros dos grupos é algo que seus integrantes constroem conversando e trabalhando juntos. Essa aprendizagem apresenta-se relevante na atualidade considerando-se as inovações científicas e tecnológicas que propiciam uma quantidade cada vez maior de informações que a comunidade acadêmica deve manusear para transformar em conhecimentos relevantes (Torres et. al., 2004). Partindo desses pressupostos, as tecnologias moveis podem servir de suporte à aprendizagem colaborativa como ferramenta para provocar um maior interesse por conteúdos desenvolvidos. O Whatsapp apresenta uma interface favorável para propiciar uma interatividade necessária ao desenvolvimento da aprendizagem colaborativa durante a utilização do aplicativo, contribuindo para que os alunos integrantes dos grupos de Whatsapp sejam responsáveis por sua aprendizagem.

\section{Mapas Conceituais e Links Cognitivos}

Conforme Moreira e Buchweitz (1987), mapas conceituais são diagramas que procuram refletir a organização conceitual da estrutura cognitiva de um indivíduo sobre um dado assunto. São organizações gráficas dos conhecimentos, que procuram identificar e relacionar os conceitos existentes na estrutura cognitiva do indivíduo, formando proposições. Um mapa conceitual fornece uma demonstração visual das relações entre os conceitos e as proposições.

Os mapas conceituais podem representar as atividades do currículo de um curso, os conteúdos de uma disciplina ou também podem ser utilizados na avaliação significativa de conteúdos apresentados, como organizadores de conhecimentos prévios ou subsunçores, como organizadores prévios de sala de aula, etc. (Novak, 2005). Nesse contexto, a aprendizagem significativa de Ausubel fornece os princípios teóricos para a elaboração dos mapas conceituais, visto que os conceitos devem ser selecionados considerando suas relevâncias de acordo com conteúdos abordados. A proposição surge a partir da ligação de dois ou mais conceitos através de palavras de ligação (Novak, 2005). Conforme Moreira (2011), não havendo a ligação dos conceitos na estrutura cognitiva do indivíduo, resultará em uma aprendizagem mecânica ou por memorização, ou seja, uma aprendizagem que estabelece pouca, uma ou nenhuma relação dos novos conceitos com aqueles pré-existentes. Ausubel (1980) considera esse tipo de aprendizagem literal e arbitrária, o que propicia uma maior volatilidade dada a falta de concatenação entre os conceitos. 
A partir dos estudos realizados por Nunes (2020), em que considera como link cognitivo as inter-relações estabelecidas por dois ou mais conceitos apreendidos pelo estudante, gerando um novo conhecimento, consegue-se vislumbrar os conteúdos derivados de ambientes colaborativos com potencial significativo, desde que atendam aos pressupostos de Ausubel et al (1980). Um link cognitivo estabelece as relações "mais fortes" em um mapa conceitual, ou seja, são as proposições mais importantes para o processo de aprendizagem observadas nos caminhos formados pelos conceitos e as proposições (Nunes, 2020). Ainda, de acordo com Nunes (2020), advém o conceito de subsunçores organizados como sendo dois ou mais links concatenados, uma vez que cada link cognitivo corresponde a um subsunçor, representando, desta forma, a aprendizagem significativa proposicional, o que caracteriza a organização dos mapas conceituais. A Figura 1 apresenta esquematicamente a ideia de subsunçores organizados através de mapa conceitual.

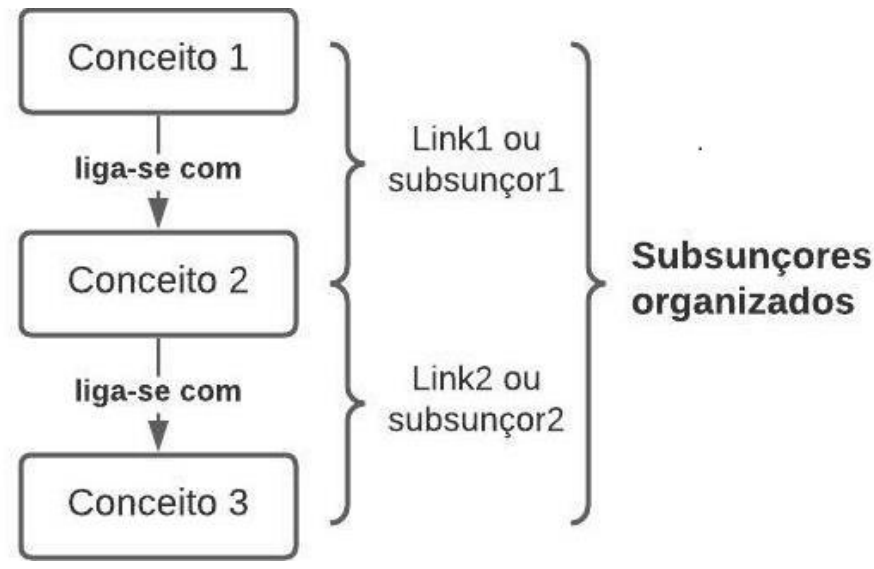

Figura 1 - Esquema de mapa conceitual

\section{Metodologia}

O grupo que participou da pesquisa cursava a disciplina de Sistema de Informação, do segundo semestre de um curso superior, da área de informática, com o total de 20 alunos, sendo 13 alunos do sexo masculino e todos eles apresentando nível médio de escolaridade, faixa etária com 15 alunos entre 19 e 24 anos, 3 na faixa de 35 a 40 anos e 1 com 50 anos. O nível de conhecimento médio de informática com 14 alunos e 7 alunos com nível alto. Essas características foram evidenciadas em pesquisa realizada através do aplicativo Google Forms, no qual coletou-se, também, a percepção dos alunos participantes em relação ao trabalho desenvolvido.

A abordagem qualitativa foi adotada nesta pesquisa, porque de acordo com Gil (2010), esse tipo de pesquisa tem o objetivo de explicar fenômenos desconhecidos em determinado(s) contexto(s), oferecendo, dessa forma, o suporte necessário ao estudo da pesquisa que investiga as aprendizagens significativas e colaborativas no contexto de uma tecnologia digital colaborativa.

De acordo com Faria (1995), a abordagem qualitativa possui relação com os mapas conceituais porque esses propiciam a busca e o aprofundamento dos significados existentes durante os processos de ensino e aprendizagem, que são características dessa abordagem. Quanto à amostra, o método utilizado para a pesquisa foi o não-probabilístico, porque existiu uma escolha deliberada do grupo a ser pesquisado, de acordo com os critérios do pesquisador (Gil, 2010). A amostra da pesquisa de 20 participantes é considerada adequada, porque Tullis e Stetson (2004) consideram válida a amostra mínima de 12 a 14 usuários em um teste de usabilidade de software, ao qual enquadra-se o aplicativo de mensagens WhatsApp. 
A coleta dos dados aconteceu após os alunos efetivarem um trabalho de respostas a um questionário com conteúdo sobre ERP, formulado a partir do artigo de Giorgia e Santos (2015), disponibilizado aos respondentes no decurso da disciplina de Sistemas de Informação. As tarefas de leitura do artigo e respostas ao questionário foram desenvolvidas totalmente através do aplicativo WhatsApp, levando os alunos a interagirem de forma colaborativa, postando suas observações e questionando os colegas, até chegarem à formatação de suas respostas.

O professor da disciplina organizou a turma de alunos em 4 grupos de 5 componentes, cada grupo gerou um grupo no WhatsApp, nos quais os alunos desenvolveram as respostas do trabalho, em 1 semana. Salienta-se a recomendação de que a única forma de comunicação deveria ocorrer através do aplicativo de mensagens, além disso, o trabalho foi realizado de forma remota, o que aumentou a importância da troca virtual entre os participantes. A análise das respostas do trabalho foi objeto da avaliação dos pesquisadores, que o fizeram procurando evidências da aprendizagem significativa nas respostas postadas nos grupos.

\section{Análise dos Resultados}

Conforme abordado, o trabalho foi desenvolvido com a utilização do aplicativo de mensagens WhatsApp, no qual os alunos trabalharam colaborativamente na formulação das respostas do questionário. Na Figura 2, observa-se um recorte da discussão em torno de uma das respostas desenvolvidas pelo Grupo 5. Pode-se observar a interação colaborativa entre dois alunos de forma crítica ao artigo, analisando-o de acordo com suas experiências, evidenciando as características da aprendizagem ativa e significativa abordada por Mill (2021). Verificou-se, também, esses mesmos pressupostos nos diálogos desenvolvidos nos 5 grupos analisados, apontando, já nessa primeira análise, o potencial do aplicativo de mensagem para a aprendizagem.

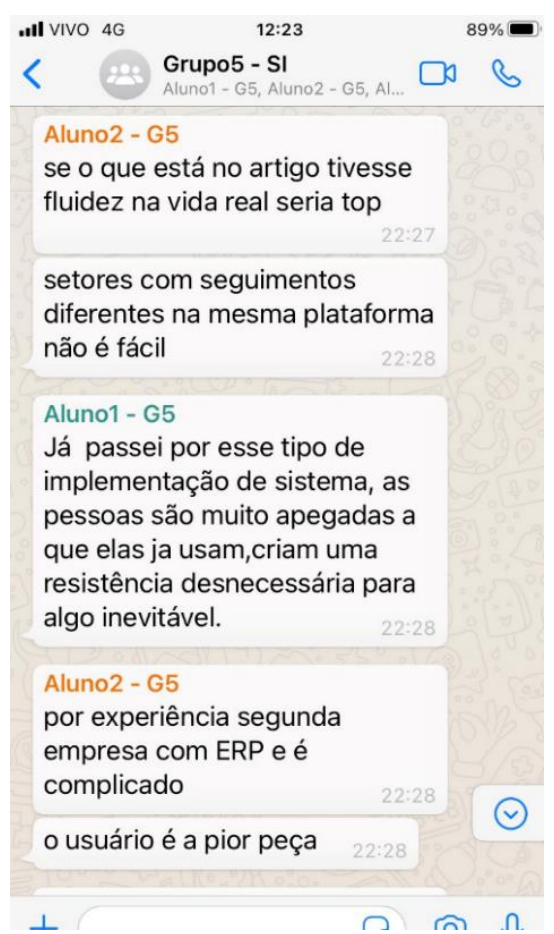

Figura2 - Recorte do WhatsApp

Buscando-se maiores evidências quanto às aprendizagens significativa e ativa consideraram-se as análises com mapas conceituais, utilizados como ferramenta para o desenvolvimento do trabalho e elaborados a partir do artigo de Giorgia e Santos (2015), que analisa um sistema de ERP implantado em uma empresa do ramo têxtil. Para tanto, 
desenvolveu-se um questionário com questões relativas a cada um dos capítulos do artigo. $\mathrm{O}$ questionário foi aplicado em cada um dos grupos pesquisados. Os resultados foram verificados analisando-se as respostas de cada um dos grupos, através da obtenção dos subsunçores organizados com a interrelação de dois ou mais links cognitivos. Porque de acordo com Faria (1995), os mapas conceituais são instrumentos importantes para o sistema de avaliação, dandolhe mais eficácia, pois focalizam aspectos da estrutura conceitual do conteúdo, ou seja, para que os mapas advindos das respostas fornecidas pelos alunos estejam bem estruturados, eles devem ter uma compreensão ampla do material estudado, discernindo os conceitos mais gerais e os vários níveis de conceitos subordinados. Assim, consegue-se estruturar e retratar de forma significativa o conteúdo de ensino através de mapas conceituais, certamente a utilização desse instrumento para avaliar o conhecimento do estudante nessa estrutura é o mais indicado (Novak, 2005).

As perguntas formuladas a partir do artigo foram:

1. Comente como as empresas procuram adequar seus processos internos de forma a concorrer competitivamente e de que maneira a organização desses processos pode influenciar positivamente em suas estruturas;

2. Considerando a implantação de um sistema de gestão $E R P$, analise as contribuições de um modelo genérico de implementação de TI e suas consequências no ciclo de vida do sistema;

3. A metodologia utilizada no trabalho apoiou-se em tipos bem específicos. Descreva de que forma eles se relacionam através da análise do ERP da empresa;

4. O diagnóstico mostrou questões importantes, advindas do ERP implantado na empresa. Diga quais são e aponte algumas soluções; e

5. Descreva quais foram os principais problemas identificados na análise da implantação do ERP, constatados pelo estudo e que ações podem ser desenvolvidas para minorá-los.

Todas as questões foram elaboradas pelos pesquisadores, realizadas a partir dos mapas conceituais, advindos de cada um dos capítulos do artigo de Giorgia e Santos (2015). A seguir,apresenta-se a Figura 3, com o mapa conceitual da questão 2.

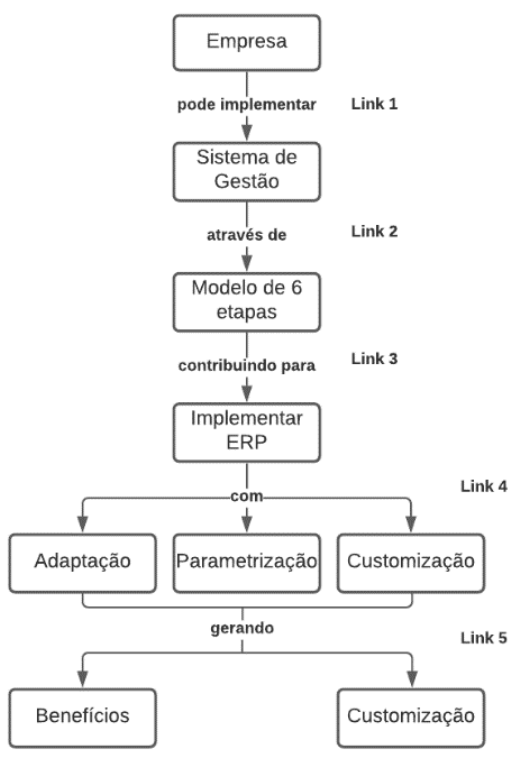

Figura3 - Mapa conceitual da questão 2 
Na Tabela 1 observam-se as questões, os links assinalados com x coletados das respostas das questões do grupo 2, que corresponderam aos links cognitivos dos mapas conceituais de cada uma das questões e os subsunçores organizados em cada uma delas.

Tabela 1 - Subsunçores organizados do grupo 2

\begin{tabular}{c|c|c|c|c|c|c}
\hline \multicolumn{7}{c}{ Grupo 2 } \\
\hline Questões & Link1 & Link2 & Link3 & Link4 & Link5 & SO \\
\hline 1 & $\mathrm{x}$ & & $\mathrm{x}$ & $\mathrm{x}$ & $\mathrm{x}$ & 3 \\
\hline 2 & $\mathrm{x}$ & & $\mathrm{x}$ & $\mathrm{x}$ & $\mathrm{x}$ & 3 \\
\hline 3 & $\mathrm{x}$ & $\mathrm{x}$ & $\mathrm{x}$ & $/ / / / / / / / / / / / / / / / / / / / / / / / / / / / / / / / / / / /$ & 3 \\
\hline 4 & $\mathrm{x}$ & $\mathrm{x}$ & $\mathrm{x}$ & $/ / / / / / / / / / / / / / / / / / / / / / / / / / / / / / / / / / /$ & 3 \\
\hline 5 & & $\mathrm{x}$ & $\mathrm{x}$ & $\mathrm{x}$ & $/ / / / / / / / / / / / / / /$ & 3 \\
\hline
\end{tabular}

Observação: A parte assinalada com hachuras (//I////) na tabela indica a inexistência de links cognitivos nas respectivas questões.

Verifica-se, através da Tabela 1, que em cada uma das questões o grupo 2 obteve 3 subsunçores organizados. Nas questões 1 e 2 nota-se que o Linkl aparece não concatenado com o Link2 demonstrando que os conceitos ou subsunçores de competitividade da questão 1 e o de empresa da questão 2 podem existir na estrutura cognitiva dos alunos do grupo 2, porém, não se relacionaram com os conceitos a eles subjacentes e, dessa forma, não acontecendo a organização dos subsunçores entre os Links 1 e 2, o que demonstra que não ocorreu a aprendizagem significativa entre essas 2 proposições, caracterizando, desta forma, apenas a aprendizagem mecânica desses dois Links. Entretanto, nessas mesmas questões, aconteceu a concatenação entre os Links 3, 4 e 5, organizando os subsunçores e efetivando a aprendizagem significativa entre eles, gerando novos conceitos. Da forma análoga, pode-se analisar os Links cognitivos 2, 3 e 4 da questão 5 .

A Figura 4 exemplifica visualmente os links concatenados através do mapa conceitual da questão 2, somente com os subsunçores organizados.

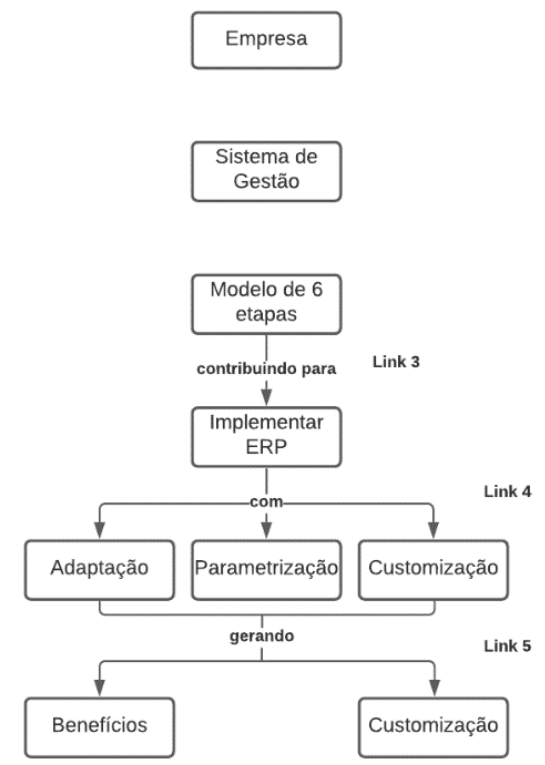

Figura 4 - Mapa conceitual da questão 2 concatenado 
A Tabela 2 mostra o total de subsunçores organizados por cada um dos grupos:

Tabela 2 - Subsunçores organizados por grupos

\begin{tabular}{c|c}
\hline Grupo & $\begin{array}{c}\text { Subsunçores } \\
\text { Organizados }\end{array}$ \\
\hline 1 & 15 \\
\hline 2 & 15 \\
\hline 3 & 19 \\
\hline 4 & 13 \\
\hline 5 & 12 \\
\hline Total & 74 \\
\hline
\end{tabular}

Considerando-se que o total de subsunçores organizados nas 5 questões é 20, observase, na Tabela 2, o Grupo 5 com o menor e o Grupo 3 com o maior número de subsunçores organizados e a média de 14,8. Esses dados são relevantes para evidenciar a aprendizagem significativa entre os grupos, pois a média equivale a uma "nota" igual a 7,4, significando que os grupos conseguiram organizar $+-75 \%$ dos subsunçores definidos através dos mapas conceituais de cada uma das questões.

A efetividade da organização dos subsunçores permite considerar que a utilização de mapas conceituais como ferramenta avaliativa evidenciou a aprendizagem ativa no trabalho desenvolvido utilizando o WhatsApp como tecnologia digital colaborativa, uma vez que os links cognitivos concatenados mostraram os atributos necessários para a aprendizagem significativa.

Ademais, verificou-se que o WhatsApp utilizado como ferramenta pedagógica colaborativa pode ser uma interface importante para a aprendizagem ativa, conforme demonstram os subsunçores organizados com a análise dos mapas conceituais dos grupos estudados. É importante salientar a aprovação dos estudantes com relação ao aplicativo de mensagens, ao responderem uma pesquisa de satisfação através do Google Forms. A seguir, algumas impressões dos alunos:

\footnotetext{
"Achei ótimo utilizar o WhatsApp como ferramenta para realizar o trabalho"; "Acho que o WhatsApp é uma boa ferramenta de comunicação entre grupos escolares e para facilitar o desempenho dos alunos. Na minha opinião, o WhatsApp deve sim ser utilizado e explorado para fins acadêmicos";

"A utilização do WhatsApp é tranquila, pois uso regularmente no trabalho, pois a comunicação e o retorno das soluções são quase imediatos. Sendo que várias pessoas podem interagir ao mesmo tempo".
}

Através dos comentários percebe-se que os estudantes possuíam familiaridade com o WhatsApp, o que colaborou para o desenvolvimento do trabalho e, também, para opinarem a respeito de sua utilidade pedagógica. Assim, pode-se afirmar que esse fato contribuiu para a aprendizagem ativa, pois através dessa interface colaborativa os elementos dos grupos demonstraram ser protagonistas do próprio processo de construção do conhecimento (Mill, 2021), o que caracteriza, também, a aprendizagem significativa.

\section{Considerações Finais}

As tecnologias digitais móveis desafiam as instituições a sair do ensino tradicional centrado no professor como transmissor do conhecimento, para uma aprendizagem mais ativa e integradora com a participação efetiva dos estudantes, através de momentos presenciais e a distância, criando vínculos pessoais e afetivos, mesmo que de forma virtual, pode-se, então, apropriar boa parte do tempo com formas ativas de aulas. Essas tecnologias podem, quando V. $19 \mathrm{~N}^{\mathrm{o}} 2$, Dezembro, 2021 RENOTE 
bem estruturadas, provocarem mudanças metodológicas nos processos de ensino e aprendizagem, dada a interação on e offline, favorecendo à aprendizagem colaborativa (Moran et al, 2013).

As práticas pedagógicas voltadas para o envolvimento ativo dos estudantes de forma que sejam protagonistas de sua aprendizagem, estimulados por métodos que favoreçam esse processo, embasam a aprendizagem ativa (Valente, Almeida e Geraldi, 2017). Logo, a aprendizagem significativa enquanto suporte à aprendizagem ativa contribui para o envolvimento efetivo dos aprendentes, através das conexões estabelecidas entre conceitos novos e conceitos já estabelecidos em suas estruturas cognitivas. Esse suporte proporcionado pelo estabelecimento de significados torna-se relevante para o estudo colaborativo de conteúdos específicos (Paiva et al, 2016). Sendo assim, as tecnologias digitais colaborativas podem, se estruturadas adequadamente, contribuir como interface pedagógica para a aprendizagem significativa, conforme visto neste trabalho, através das análises efetivadas.

Considerando-se as tecnologias digitais colaborativas como ambientes de aprendizagem colaborativa, concluiu-se que esses atuam como instrumentos cognitivos, ajudando os alunos a aprender de maneira significativa e ativa. Da mesma forma, servem para ampliar, potencializar e reorganizar as capacidades dos estudantes, conduzindo-os a uma aprendizagem significativa (Filho e Medina, 2017). Assim sendo, os resultados encontrados pela pesquisa, acompanhada de suas análises, apontaram para a interlocução entre as aprendizagens significativa e ativa, o que estimula novos estudos, aproveitando as experiências aqui alcançadas, através dessas aprendizagens aplicadas aos ambientes colaborativos.

\section{Referências}

AUSUBEL, D.; NOVAK, J.; HANESIAN, H. Psicologia Educacional. Rio de Janeiro: Interamericana, 1980.

FARIA, W. Mapas Conceituais: aplicações ao ensino, currículo e avaliação. São Paulo: E.P.U., 1995.

GIL, A. C. Como elaborar projetos de pesquisa. 5 ed. São Paulo: Atlas, 2010. GIORGIA M., SANTOS W. S. Sistema ERP: Estudo Exploratório Numa Empresa do Setor Têxtil do Agreste Pernambucano. Revista Brasileira de Gestão e Inovação - Brasilian Journal of Management \& Innovation, v. 2, n. 3, 2015.

KURTZ, R.; SOARES, D.M.T.; FERREIRA, J.B; FREITAS, A.S. de; SILVA, J. F. da. Fatores de impacto na atitude e na intenção do uso do m-learning: um teste empírico. Revista eletrônica de administração, v. 21, n. 1, p. 27-56, 2015.

LAAL, M.; LAAL, M. Collaborative learning: what is it?. Procedia-Social and Behavioral Sciences, v. 31, p. 491-495, 2012.

MEDINA N. O., FILHO P. J. F. Análise da Aprendizagem Significativa em Ambientes de Escrita colaborativa Apoiada por Computador. Revista Brasileira de Informática na Educação, v.15, n. 2, 2017. Disponível em: $<$ https://www.researchgate.net/publication/277738397_Analise_da_Aprendizagem_Significat iva_em_Ambientes_de_Escrita_Colaborativa_Apoiada_por_Computador>. Acesso em: 13 jun. 2021. 
MILL, D. Sobre a aprendizagem ativa e significativa na cultura digital. São Carlos: SEaD, UFSCar, 2021.

MORAN, J. M., MAZETTO, T., BERENS M. A. Novas Tecnologias e Mediação

Pedagógica. Campinas: Papirus, 2013.

MOREIRA, M. e BUCHWEITZ, B. Mapas Conceituais: instrumentos didáticos de avaliação e de análise de currículo. São Paulo: Moraes, 1987.

MOREIRA, M. A. Aprendizagem significativa: um conceito subjacente.

Aprendizagem Significativa em Revista, v. 1, n. 3, p. 25-46, 2011. Disponível em: <http://www.if.ufrgs.br/asr/artigos/Artigo_ID16/v1_n3_a2011.pdf>. Acesso em: 25 jul. 2021.

NOVAK, J. D. Learning, Creating, and Using Knowledge: concept maps as facilitative tools is schools and corporation. Lawrence Erlbaum Associates, 2005.

NUNES, S. C. A Aprendizagem Significativa em Portal Educacional: Uma análise dos conhecimentos prévios. 1. Ed. Porto Alegre: Amazon, 2020. V. 1. 120p.

PAIVA, M. R. F.; PARENTE, J. R. F.; BRANDÃO, I. R.; QUEIROZ, A. H. B. Metodologias ativas de ensino-aprendizagem: revisão integrativa. SANARE, v. 15 n. 2, p. 145-153, 2016. Disponível em: $<$ https://sanare.emnuvens.com.br/sanare/article/download/1049/59 5>. Acesso em: 25 jun. 2021.

SANTOS, C. M. R. G.; FERRARI, M. A. (Orgs.). Aprendizagem ativa: contextos e experiências em comunicação. Bauru: Universidade Estadual Paulista, 2017.

SMIT, I.; GOEDE, R. WhatsApp with BlackBerry; Can messengers (BBM) be MXit?. Proceedings of the 14th annual conference on world wide web applications. Cape Town, South Africa: Cape Peninsula University of Technology, 2012. Disponível em: <http://hdl.handle.net/10394/13628>. Acesso em: 02 ago. 2021.

TORRES, P. L.; ALCANTARA, P.; IRALA, E. A. F. Grupos de consenso: uma proposta de aprendizagem colaborativa para o processo de ensino e aprendizagem. Revista diálogo educacional, v. 4, n. 13, p. 129-145, 2004.

TULLIS, T. S.; STETSON, J. N. A Comparison of Questionnaires for Assessing Website Usability, 2004. Disponível em:

$<$ http://home.comcast.net/ tomtullis/publications/UPA2004TullisStetson.pdf >. Acesso em: 10 jul. 2021.

VALENTE, J. A.; ALMEIDA, M. E. B.; GERALDINI, A. F. S. Metodologias ativas: das concepções às práticas em distintos níveis de ensino. Revista Diálogo

Educacional, v. 17, n. 52, p. 455-478, abr./jun. 2017. Disponível em: $<$ https://periodicos.pucpr.br/index.php/dialogoeducacional/article/view/9900/12386>. Acesso em: 25 jun. 2021. 